\title{
Combined Therapy with Peritoneal Dialysis and Hemodialysis: A Multicenter Retrospective Observational Cohort Study in Japan
}

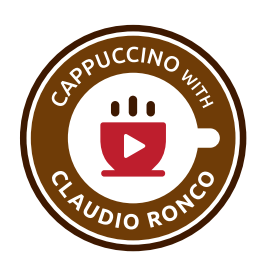

Yukio Maruyama ${ }^{a, b}$ Keitaro Yokoyama ${ }^{a, b}$ Masaaki Nakayamac Chieko Higuchi ${ }^{d}$

Tsutomu Sanakad ${ }^{d}$ Yoshihide Tanaka $^{e}$ Ken Sakai ${ }^{\text {S Sonoo Mizuirie }}$

Yasushi Otsuka $^{a}$ Satoru Kuriyama ${ }^{a}$ Teruhiko Maeba ${ }^{f}$ Hideaki Iwasawag $^{g}$

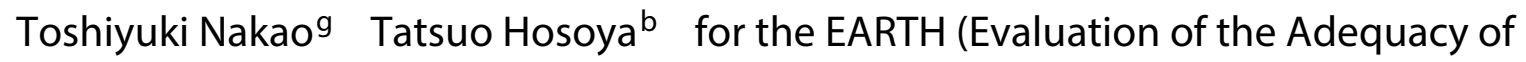

Renal replacement THerapy) study group

${ }^{a}$ Division of Nephrology and Hypertension, Department of Internal Medicine, ${ }^{b}$ Department of Pathophysiology and Therapy in Chronic Kidney Disease, The Jikei University School of Medicine, Tokyo, 'Department of Nephrology and Hypertension, Fukushima Medical University School of Medicine, Fukushima, d'Division of Nephrology and Blood Purification, Department of Internal Medicine, Tokyo Women's Medical University Medical Center East, e Department of Nephrology, Toho University School of Medicine, Tokyo, ${ }^{f}$ Asao Kidney Clinic, Kanagawa, and ${ }^{9}$ Department of Nephrology, Tokyo Medical University, Tokyo, Japan

\section{Key Words}

$\beta 2$ microglobulin - Dialysate-to-plasma ratio of creatinine $(\mathrm{D} / \mathrm{P} \mathrm{Cr}) \cdot$ Dialysis adequacy $\cdot$ Hemodialysis · Peritoneal dialysis · Peritoneal equilibration test (PET) · Residual renal function

\footnotetext{
Abstract

Background/Aims: Combining peritoneal dialysis (PD) and hemodialysis (HD) has been common treatment option in Japan. Methods: In this retrospective, multicenter, observational study, the clinical characteristics and outcomes of 104 patients (57 \pm 11 years, males $72 \%$ ) who had switched from PD alone to combined therapy with PD and HD were studied. Clinical parameters were measured at baseline and after 3 months of combined therapy. Results: At baseline, urine volume, dialysate-to-plasma ratio of creatinine (D/P Cr), and total $\mathrm{Kt} / \mathrm{V}$ were $150 \mathrm{ml} /$ day (range: $0-2,000 \mathrm{ml} /$ day), $0.67 \pm$
}

0.11 , and $1.8 \pm 0.4$, respectively. During the first 3 months of combined therapy, body weight, urine volume, serum creatinine level, and D/P Cr decreased, whereas hemoglobin levels increased. Conclusions: In patients where PD does not result in acceptable outcomes, combined therapy with PD and HD may have potential benefits in terms of dialysis adequacy and hydration status.

Video Journal Club "Cappuccino with Claudio Ronco" at http://www.karger.com/?doi=368389@ @2014 S. Karger AG, Basel

\section{Introduction}

Although peritoneal dialysis (PD) is recommended as a first-line treatment for end-stage renal disease (ESRD) [1], the efficiency of PD gradually worsens over time. In Japanese PD patients, 5-year technique survival was esti-

\section{KARGER}

E-Mail karger@karger.com www.karger.com/bpu
(C) 2014 S. Karger AG, Basel

0253-5068/14/0382-0149\$39.50/0 
mated to be $70 \%$, and the most common reasons for this technique to become a failure are inadequate dialysis and/ or ultrafiltration failure [2]. Treatment options for these patients include increasing the dose of $\mathrm{PD}$, switching to hemodialysis (HD) or starting a combined therapy with PD and HD.

Combined therapy was first introduced in Japan in the 1990s, and from then on, it has rapidly gained popularity. It is a treatment choice for PD patients who cannot achieve adequate fluid and/or solute removal by $\mathrm{PD}$ alone. Generally, it comprises five to six days of PD combined with one HD session per week. In 2012, approximately 1,900 patients ( $20 \%$ of all PD patients) were on this therapy in Japan (Japanese Society for Dialysis Therapy, unpublished data).

Although there have been several published reports, most studies till date were limited by the population size or by being single-center studies [3-9]. To better evaluate the clinical benefit of this modality, we established the Evaluation of the Adequacy of Renal replacement THerapy (EARTH) Study Group. This paper presents the clinical outcomes of more than 100 patients on combined therapy across nine centers in Japan.

\section{Materials and Methods}

\section{Subjects}

Inclusion criteria of this retrospective, multicenter, observational cohort study was switching from PD alone to combined therapy with PD and HD. We identified 104 patients (75 males and 29 females; mean age at the start of combined therapy: $57 \pm$ 11) from nine facilities in Japan, including: Jikei University Hospital and three branch hospitals, Kawaguchi Municipal Medical Center; Toho University Omori Medical Center; Tokyo Medical University Hospital; Tokyo Women's Medical University Medical Center East, and Saiseikai Central Hospital. All 104 patients switched from PD alone to combined therapy between November 1998 and November 2009. The study was conducted in accordance with the Declaration of Helsinki. Table 1 shows baseline patient characteristics. The median duration of PD at the start of the combined therapy was 37 months (range: 2-250 months) (fig. 1).

\section{Combined Therapy Regimens}

In general, PD was not carried out on the day of an HD session, and 57 patients $(61 \%)$ also did not perform PD on another day, which was defined as a 'PD holiday'. Twenty-six patients $(26 \%)$ used automated $\mathrm{PD}$, and 41 patients (41\%) used icodextrin solution. Regarding the HD prescription, 81 patients $(81 \%)$ received HD once a week for $4 \mathrm{~h}$. Only two patients (2\%) received HD twice a week. High-flux membranes were used for all HD sessions. Blood samples were drawn from the arterial line at the start of the HD treatment.
Table 1. Baseline patient characteristics $(\mathrm{n}=104)$

\begin{tabular}{ll}
\hline Category & Results (\%/range) \\
\hline Sex, \% & \\
$\quad$ Male & $75(72)$ \\
Female & $29(28)$ \\
Age at the start of combined therapy, years & $57 \pm 11$ \\
Cause of ESRD & $54(52)$ \\
CGN & $29(28)$ \\
Diabetic nephropathy & $6(6)$ \\
Nephrosclerosis & $2(2)$ \\
PKD & $13(12)$ \\
Others or unknown & $23(22)$ \\
Comorbidities & $10(10)$ \\
Ischemic heart disease & $3(3)$ \\
Cerebrovascular disease & \\
Malignant disease & $37(2-250)$ \\
Duration of PD at the start of combined & \\
$\quad$ therapy, months & \\
\hline
\end{tabular}

$\mathrm{PD}=$ Peritoneal dialysis; $\mathrm{CGN}=$ chronic glomerulonephritis; $\mathrm{PKD}=$ polycystic kidney disease.

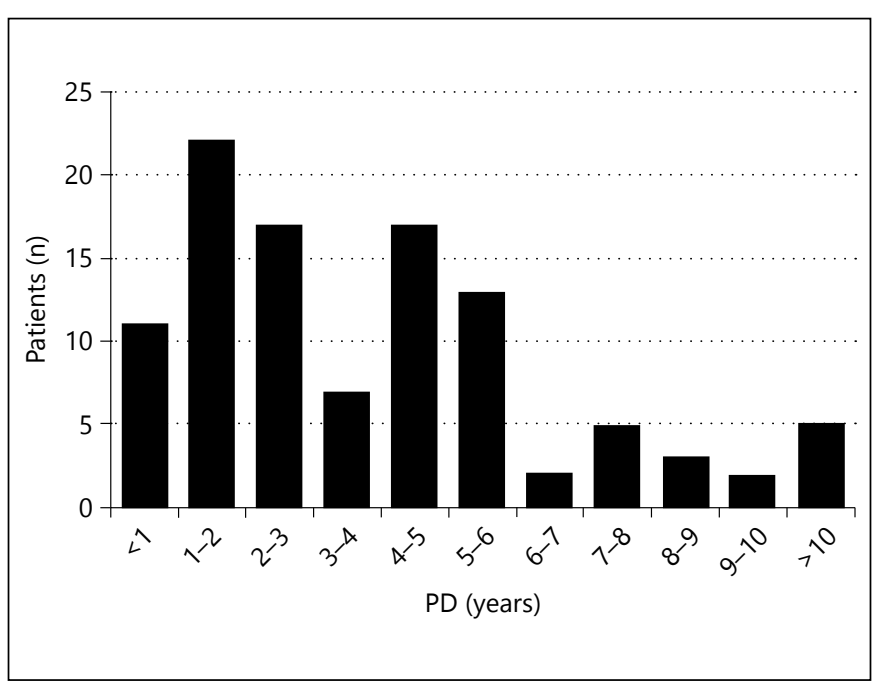

Fig. 1. Distribution of the duration of PD at the start of combined therapy.

\section{Study Outcomes}

We compared the clinical and laboratory data on dialysis adequacy and volume status just before switching therapy with the data collected 3 months later. Study outcomes included changes in body weight, blood pressure, urine volume, peritoneal ultrafiltration volume, blood urea nitrogen $(\mathrm{BUN})$, creatinine $(\mathrm{Cr}), \beta 2 \mathrm{mi}-$ croglobulin, and hemoglobin. Additionally, changes in dialysateto-plasma ratio of creatinine ( $\mathrm{D} / \mathrm{P} \mathrm{Cr})$, obtained from a peritoneal equilibration test (PET) were examined. The total Kt/V and total 
Table 2. Clinical and biochemical parameters before and after 3 months of combined therapy in 104 PD patients

\begin{tabular}{lccr}
\hline Parameters & \multicolumn{2}{c}{ Results } & p value \\
\cline { 2 - 3 } & $\begin{array}{l}\text { before combined } \\
\text { therapy }\end{array}$ & $\begin{array}{l}\text { after } 3 \text { months } \\
\text { of combined therapy }\end{array}$ \\
\hline Body weight, kg & $64.5 \pm 14.8$ & $63.4 \pm 14.2$ & $<0.01$ \\
Systolic BP, mm Hg & $144 \pm 22$ & $143 \pm 20$ & 0.83 \\
Diastolic BP, mm Hg & $80 \pm 14$ & $81 \pm 14$ & 0.76 \\
Urine volume, ml/day & $150(0-2,000)$ & $75(0-1,900)$ & $<0.01$ \\
Amount of the PD solution, ml/day & $8,200 \pm 1,600$ & $8,100 \pm 1,600$ & 0.38 \\
Peritoneal ultrafiltration volume, ml/day & $1,000(-500$ to 2,350$)$ & $950(-1,000$ to 2,700$)$ & 0.46 \\
BUN, mg/dl & $59.2 \pm 14.6$ & $57.2 \pm 13.5$ & 0.13 \\
Cr, mg/dl & $12.9 \pm 3.4$ & $12.3 \pm 3.0$ & $<0.01$ \\
$\beta 2$ microglobulin, mg/l & $34.4 \pm 7.2$ & $33.7 \pm 7.0$ & 0.23 \\
Hgb, g/dl & $8.7 \pm 1.5$ & $10.3 \pm 1.3$ & $<0.01$ \\
D/P Cr & $0.67 \pm 0.11$ & $0.61 \pm 0.13$ & $<0.01$ \\
Total Kt/V & $1.8 \pm 0.4$ & n.a. & n.a. \\
Total weekly Ccr, ml/min/1.73 m ${ }^{2}$ & $49.9 \pm 13.1$ & & \\
\hline
\end{tabular}

$\mathrm{PD}=$ Peritoneal dialysis; $\mathrm{HD}=$ hemodialysis; $\mathrm{BP}=$ blood pressure; $\mathrm{BUN}=$ blood urea nitrogen; $\mathrm{Cr}=\mathrm{creati}$ nine; $\mathrm{Hgb}=$ hemoglobin; $\mathrm{D} / \mathrm{P} \mathrm{Cr}=$ dialysate-to-plasma ratio of creatinine; $\mathrm{Ccr}=$ creatinine clearance; $\mathrm{n} . \mathrm{a} .=\mathrm{not}$ applicable.

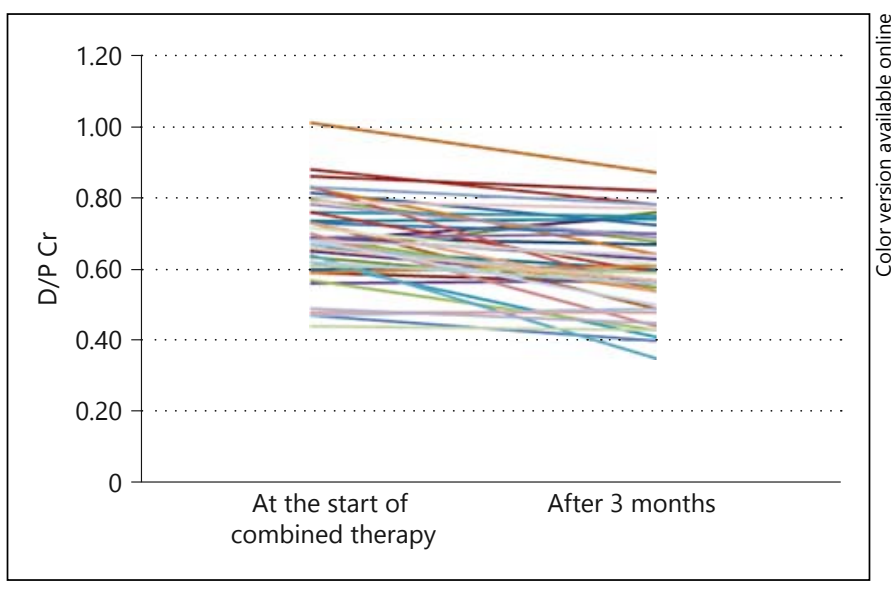

Fig. 2. Change in dialysate-to-plasma ratio of creatinine (D/P Cr).

weekly creatinine clearance rate (Ccr) just before switching therapy were calculated using the guidelines from the Japanese Society for Dialysis Therapy [10].

\section{Statistical Analysis}

The changes in parameters between the start of the combined therapy and 3 months later were evaluated using a paired $t$ test or the Wilcoxon signed-rank test when appropriate. Data are presented as mean $\pm \mathrm{SD}$ or medians and ranges when appropriate. Statistical significance is defined as being $\mathrm{p} \leq 0.05$. All statistical analyses were performed using JMP, version 9.0 for Windows (SAS Institute Inc., Cary, N.C., USA).

Combined PD and HD in Japan

\section{Results}

At the start of combined therapy, urine volume, $\mathrm{D} / \mathrm{P}$ $\mathrm{Cr}$, total $\mathrm{Kt} / \mathrm{V}$, and total weekly Ccr were $150 \mathrm{ml} /$ day (range: $0-2,000 \mathrm{ml} / \mathrm{day}$ ), $0.67 \pm 0.11,1.8 \pm 0.4$, and $49.9 \pm$ $13.1 \mathrm{ml} / \mathrm{min} / 1.73 \mathrm{~m}^{2}$, respectively (table 2). The results of the comparative analysis between the start of the combined therapy and 3 months later are also shown in table 2 . Body weight decreased from $64.5 \pm 14.8 \mathrm{~kg}$ to $63.4 \pm 14.2$ $\mathrm{kg}(\mathrm{p}<0.01)$. Neither systolic nor diastolic blood pressure changed significantly. Urinary volume decreased, whereas the peritoneal ultrafiltration volume did not change significantly. The mean serum creatinine levels decreased significantly from $12.9 \pm 3.4 \mathrm{mg} / \mathrm{dl}$ to $12.3 \pm 3.0 \mathrm{mg} / \mathrm{dl}$ (p < 0.01 ). Although BUN and $\beta 2$ microglobulin levels tended to decrease, these decreases were not significant. Hemoglobin levels increased from $8.7 \pm 1.5 \mathrm{~g} / \mathrm{dl}$ to $10.3 \pm 1.3 \mathrm{~g} /$ $\mathrm{dl}(\mathrm{p}<0.01)$. D/P Cr decreased significantly from $0.67 \pm$ 0.11 to $0.61 \pm 0.13(\mathrm{p}<0.01)$ (fig. 2$)$.

\section{Discussion}

In this multicenter retrospective observational cohort study, the clinical outcomes of combined therapy with PD and HD in Japan were evaluated. At the start of the combined therapy, the median PD duration was approx- 
imately 3 years, which is similar according to the previous reports [3-6]. In the current study, body weight decreased following the initiation of combined therapy, suggesting that overhydration was a main cause for starting this therapy, whereas there was only a small change in solute clearance, including $\mathrm{Kt} / \mathrm{V}$ and weekly $\mathrm{Ccr}$, and circulating $\beta 2$ microglobulin was not increased at baseline. During 3 months of combined therapy, both hydration status (represented by body weight and hemoglobin) and dialysis adequacy (represented by serum creatinine levels) were significantly improved as indicated in many previous reports $[3,5,6,9]$. On the other hand, the change in serum $\beta 2$ microglobulin levels did not reach significance. Unlike in $\mathrm{PD}$, serum $\beta 2$ microglobulin in patients receiving HD changes dramatically over a week, and we usually measure its highest value at the start of an HD session. Because the serum $\beta 2$ microglobulin level was associated with mortality among HD patients [11], and was an independent risk factor for encapsulating peritoneal sclerosis (EPS) [12], the $\beta 2$ microglobulin level is an important marker in the management of dialysis.

D/P Cr obtained by PET was dramatically decreased during this period, and this finding was also confirmed in our previous report [3]. There are several possible explanations for this. First, combined therapy could limit further deterioration of the peritoneal membrane by decreasing exposure to glucose and elimination of uremic toxins. Not only cumulative glucose exposure [13], but also uremia per se [14] are associated with structural alternations in the peritoneum. Second, peritoneal rest could have a positive impact on peritoneal function. Third, histological improvement of peritoneal edema secondary to improvement of fluid status might lead to the reduction in $\mathrm{D} / \mathrm{P} \mathrm{Cr}$.

Combined therapy with $\mathrm{PD}$ and HD may also give rise to some concerns. Considering that the preservation of $\mathrm{RRF}$ is one of the most important factors for selecting standard PD, first it is a troubling observation that the urine volume decreased, whereas the peritoneal ultrafiltration volume did not change in the current study. Since there have been many reports showing the association between RRF and mortality among PD patients $[15,16]$, a decline in RRF by combined therapy might lead to a poor outcome. Second, although combined therapy would increase the overall duration of PD treatment, this could have some drawbacks. For example, it is well known that prolonged PD duration is an independent risk factor for EPS, Yamamoto et al. [17] reported that the cut-off point of PD duration for the development of EPS was approximately 10 years.
There are several limitations to the present study. First, we did not have a control group, and the observational design allows only limited conclusions. Second, data of erythropoietin-stimulating agents (ESA) and antihypertensive drug were not collected, making the interpretation of hemoglobin and blood pressure data difficult. Third, we did not evaluate the nutritional status. Because both body weight and creatinine levels decreased during 3 months of combined therapy, we cannot deny the possibility of impaired nutritional status with decrease in muscle mass. Fourth, there was no universal consensus on the indication for introduction or cessation of the combined therapy. To address these and other unanswered questions, a multicenter, prospective, observational study of patients switching from PD to combined therapy is planned.

\section{Conclusions}

In this large retrospective observational cohort study, both overhydration and inadequate dialysis appear to have been improved by switching from PD alone to combined therapy with PD and HD. Further studies are needed to clarify the indications for and consequences of switching from standard PD alone to combined therapy.

\section{Acknowledgements}

The authors gratefully acknowledge EMEDITS (www.emedits. com) for editorial support in preparing this manuscript.

\section{Disclosure Statement}

The authors have no conflicts of interest to disclose.

References

1 Chaudhary K, Sangha H, Khanna R: Peritoneal dialysis first: rationale. Clin J Am Soc Nephrol 2011;6:447-456.

2 Nakamoto H, Kawaguchi Y, Suzuki H: Is technique survival on peritoneal dialysis better in Japan? Perit Dial Int 2006;26:136-143.

$\checkmark 3$ Matsuo N, Yokoyama K, Maruyama Y, Ueda Y, Yoshida H, Tanno Y, Yamamoto R, Terawaki $\mathrm{H}$, Ikeda $\mathrm{M}$, Hanaoka $\mathrm{K}$, Yamamoto $\mathrm{H}$, Ogura M, Watanabe S, Kimura Y, Hosoya T: Clinical impact of a combined therapy of peritoneal dialysis and hemodialysis. Clin Nephrol 2010;74:209-216.

4 Moriishi M, Kawanishi H, Tsuchiya S: Impact of combination therapy with peritoneal dialysis and hemodialysis on peritoneal function. Adv Perit Dial 2010;26:67-70. 
5 Kawanishi H, Hashimoto Y, Nakamoto H, Nakayama M, Tranaeus A: Combination therapy with peritoneal dialysis and hemodialysis. Perit Dial Int 2006;26:150-154.

$\checkmark 6$ Suzuki H, Hoshi H, Inoue T, Kikuta T, Tsuda M, Takenaka T: Combination therapy with hemodialysis and peritoneal dialysis. Contrib Nephrol 2012;177:71-83.

$\checkmark 7$ McIntyre CW: Bimodal dialysis: an integrated approach to renal replacement therapy. Perit Dial Int 2004;24:547-553.

-8 Agarwal M, Clinard P, Burkart JM: Combined peritoneal dialysis and hemodialysis: our experience compared to others. Perit Dial Int 2003;23:157-161.

$\checkmark 9$ Kawanishi H, Moriishi M, Tsuchiya S: Evaluation of dialysis dose during combination therapy with peritoneal dialysis and hemodialysis. Adv Perit Dial 2007;23:135-139.

10 2009 Japanese Society for Dialysis Therapy guidelines for peritoneal dialysis. Ther Apher Dial 2010;14:489-504.
11 Cheung AK, Rocco MV, Yan G, Leypoldt JK, Levin NW, Greene T, Agodoa L, Bailey J, Beck GJ, Clark W, Levey AS, Ornt DB, Schulman G, Schwab S, Teehan B, Eknoyan G: Serum beta-2 microglobulin levels predict mortality in dialysis patients: results of the HEMO study. J Am Soc Nephrol 2006;17:546-555.

12 Yokoyama K, Yoshida H, Matsuo N, Maruyama Y, Kawamura Y, Yamamoto R Hanaoka K, Ikeda M, Yamamoto H, Nakayama M, Kawaguchi Y, Hosoya T: Serum beta2 microglobulin (beta2MG) level is a potential predictor for encapsulating peritoneal sclerosis (EPS) in peritoneal dialysis patients. Clin Nephrol 2008;69:121-126.

13 Witowski J, Jorres A, Korybalska K, Ksiazek K, Wisniewska-Elnur J, Bender TO, PasslickDeetjen J, Breborowicz A: Glucose degradation products in peritoneal dialysis fluids: do they harm? Kidney Int Suppl 2003;S148S151.

$>14$ Williams JD, Craig KJ, Topley N, Von Ruhland C, Fallon M, Newman GR, Mackenzie RK, Williams GT: Morphologic changes in the peritoneal membrane of patients with renal disease. J Am Soc Nephrol 2002;13:470479.
5 Bargman JM, Thorpe KE, Churchill DN: Relative contribution of residual renal function and peritoneal clearance to adequacy of dialysis: a reanalysis of the CANUSA study. J Am Soc Nephrol 2001;12:2158-2162.

16 Paniagua R, Amato D, Vonesh E, CorreaRotter R, Ramos A, Moran J, Mujais S: Effects of increased peritoneal clearances on mortality rates in peritoneal dialysis: ADEMEX, a prospective, randomized, controlled trial. J Am Soc Nephrol 2002;13: 1307-1320.

17 Yamamoto R, Otsuka Y, Nakayama M, Maruyama Y, Katoh N, Ikeda M, Yamamoto H, Yokoyama K, Kawaguchi Y, Matsushima M: Risk factors for encapsulating peritoneal sclerosis in patients who have experienced peritoneal dialysis treatment. Clin Exp Nephrol 2005;9:148-152. 